\title{
Comorbid Zolpidem Dependence and Over-the-Counter Compound Analgesic Abuse
}

\author{
Hyounwook Kim, Cheolmin Shin, Young-hoon Ko, Changsu Han \\ Department of Psychiatry, Korea University Ansan Hospital, Korea University College of Medicine, Seoul, Korea
}

\begin{abstract}
Zolpidem is a commonly prescribed hypnotic used to treat insomnia. However, its potential for abuse and dependence has recently become controversial. Although over-the-counter (OTC) medications are widely used, their abuse potential has not received much research attention. We report a case of comorbid zolpidem and OTC compound analgesic abuse. OTC analgesics may serve as gateway drugs, and physicians must be cautious about this issue, especially when prescribing hypnotics or benzodiazepines.
\end{abstract}

KEY WORDS: Zolpidem; Dependence; Over the counter drugs; Abuse.

\section{INTRODUCTION}

Zolpidem, an imidazopyridine agent, is an agonist of the $\gamma$-aminobutyric acid (GABA) $)_{A}$-receptor complex. ${ }^{1)}$ It is among the most commonly prescribed hypnotics ${ }^{2)}$ due to its rapid onset, short duration of action, relatively few side effects compared with benzodiazepines, ${ }^{3)}$ and minimal abuse and dependence potential. ${ }^{1,45}$ These advantages are thought to derive primarily from its selective binding to the $\alpha 1$ subunit-containing $G A B A_{A}$ receptor. ${ }^{6-8)}$

However, a series of studies reported that the abuse and dependence potential of zolpidem is similar to that of other benzodiazepines, ${ }^{2,9)}$ rendering the use of this medication controversial.

Over-the-counter (OTC) medications are widely used and perceived to be safer than prescription medication. $^{10,11)}$ Pain relievers are the best-selling OTC drugs, ${ }^{12)}$ and $85 \%$ of all analgesics are sold OTC. ${ }^{13)}$ There has been increasing concern about the abuse potential of OTC analgesics, but very little is known about this issue.

Received: May 17, 2017 / Revised: June 2, 2017

Accepted: June 3, 2017

Address for correspondence: Changsu Han, MD, PhD, MHS Department of Psychiatry, Korea University Ansan Hospital,

Korea University College of Medicine, 123 Jeokgeum-ro,

Danwon-gu, Ansan 15355, Korea

Tel: +82-31-412-5140, Fax: +82-31-412-5144

E-mail: hancs@korea.ac.kr

ORCID: https://orcid.org/0000-0002-4021-8907
Moreover, as patients tend to underreport OTC analgesic use, a significant proportion of individuals who abuse or are dependent on these substances may remain unidentified. ${ }^{14)}$ Several studies have reported an association between OTC medication abuse (mainly dextromethorphan) and abuse of other substances. Bryner et al. ${ }^{15)}$ reported that $20.1 \%$ of adolescent dextromethorphan abusers engaged in polysubstance abuse. Falck et al. ${ }^{16)}$ reported that adolescent dextromethorphan abusers smoked more cigarettes and marijuana, consumed more alcohol, and abused more LSD, psilocybin, and 3,4-methylenedioxymethamphetamine.

We present a case of a patient hospitalized and treated for zolpidem dependence with comorbid OTC compound analgesic abuse.

\section{CASE}

A 69-year-old female visited the outpatient psychiatric clinic at our hospital with complaints of headache, insomnia, and zolpidem overuse. The patient had been suffering from headaches and insomnia for 12 years following her husband's death. This patient started taking Panpyrin ${ }^{\circledR}$ (PR; Dong-A Pharm, Seoul, Korea), an OTC oral liquid analgesic comprising $200 \mathrm{mg}$ acetaminophen, $100 \mathrm{mg}$ etenzamide, $30 \mathrm{mg}$ caffeine dehydrate, and $2 \mathrm{mg}$ chlorpheniramine since the onset of the headaches, con-

(c) This is an Open-Access article distributed under the terms of the Creative Commons Attribution Non-Commercial License (http://creativecommons.org/licenses/by-nc/4.0) which permits unrestricted non-commercial use, distribution, and reproduction in any medium, provided the original work is properly cited. 
suming more than five bottles of PR daily.

Because the patient's headaches and insomnia persisted and worsened despite her continuous overuse of $\mathrm{PR}$, the patient visited a local psychiatric clinic 5 years before her first visit to our hospital and started taking zolpidem $10 \mathrm{mg}$ daily. Because of sustained insomnia, the patient increased her dose of zolpidem against her physician's advice. After her physician refused to renew her prescription for zolpidem, she visited a number of clinics (including non-psychiatric ones), where her prescription was filled by proxy.

Four years later, the patient was taking 50-60 mg of zolpidem daily and was experiencing confusion, sleep walking, and falling at night. The patient's family persuaded her to decrease the dose of zolpidem to $10 \mathrm{mg}$ daily, but the patient self-medicated with PR ( 2 bottles) and OTC diphenhydramine, an antihistamine agent (100-250 mg; normal dose, 25-50 mg) on a daily basis for headaches and insomnia. The patient visited the psychiatric department of our hospital and was admitted.

The patient was educated about her dependence and the risks of drug overdose, and then agreed to follow our plan to taper off of zolpidem, PR, and diphenhydramine. The patient was initially given $1 \mathrm{mg}$ clonazepam, $50 \mathrm{mg}$ quetiapine, $10 \mathrm{mg}$ zolpidem, and $30 \mathrm{mg}$ duloxetine. Zolpidem was tapered for 14 days while the quetiapine was increased to $100 \mathrm{mg}$ and $2 \mathrm{mg}$ melatonin was added. Whenever the patient did not fall asleep within 1 hour after taking her regular medication, she was allowed to take one additional 12.5-mg dose of quetiapine per day. The patient was also allowed to take $650 \mathrm{mg}$ acetaminophen up to twice daily for headache. The patient eventually maintained a fair quality of sleep without zolpidem or other additional hypnotics and was discharged on hospital day 17. The patient continued outpatient treatment, experiencing several episodes of PR and zolpidem use, each of which lasted a maximum of one month.

\section{DISCUSSION}

This report describes a vulnerable patient who abused multiple easily available substances. Her first drug of abuse was the OTC analgesic, PR, which she took in response to headaches and insomnia following the death of her husband. PR was an attractive choice as it is an OTC medication that is easy to obtain and generally considered to be safe. However, the patient's superstitious beliefs about the effect of medication led to repetitive medication overuse. Moreover, there was no opportunity to educate the patient about her medication regimen because a prescription is not required for PR. Additionally, the patient did not attend to the drug label, which contains information about the recommended dosage, side effects, and so on. The patient took PR not only for headaches but also for insomnia. Abbott and Fraser ${ }^{17)}$ described two types of OTC analgesic users; one type seeks to alleviate pain, and the other type seeks to treat stress, anxiety, depression, and sleep disturbances.

Relapsing insomnia led the patient to seek and eventually abuse a more specialized medication, zolpidem. As one of the most commonly used hypnotics by both psychiatrists and non-psychiatrists, zolpidem is also relatively easily accessible. Additionally, we carefully suggest the possibility that the patient should have antidepressant effect of zolpidem which then would have contribute the patient's dependence on it. Licata et al. ${ }^{18)}$ found that zolpidem in the presence of SSRIs may have therapeutic effects in depression by elevating GABA. Ghosh et al. ${ }^{19)}$ reported a case of an old male who abused baclofen, a GABA B receptor agonist, due to its hypomanic effect. This patient's overuse of zolpidem caused obvious side effects, including nighttime confusion, sleep walking, and falls, leading the patient and her family members to seek medical and psychiatric services. While attempting to reduce her zolpidem use on her own, the patient overused another OTC medication, which contained diphenhydramine. This episode reflects the patient's vulnerability to relapse. OTC medication may serve as a gateway drug, and abusers of these substances may also be vulnerable to abusing psychotropic agents or other substances. Agley et al. ${ }^{20)}$ evaluated OTC medication as an initiator of drug-use sequences in adolescents but no other studies have been published on this subject.

Based on this case, we suggest that clinicians who prescribe hypnotics or benzodiazepines should 1) identify all medication currently prescribed for the patient; 2) thoroughly investigate the patient's history of OTC medication; and 3) consider short-term admission and readjustment of the patient's extant drug regimen according to the severity of the patient's dependence. Further longitudinal studies should address the association between OTC analgesic abuse and the abuse of other substances, 
including psychotropic agents.

\section{- Acknowledgments}

This research was supported by a grant of the Korea Health Technology R\&D Project through the Korea Health Industry Development Institute (KHIDI), funded by the Ministry of Health \& Welfare, Republic of Korea (HC15C1405).

\section{REFERENCES}

1. Holm KJ, Goa KL. Zolpidem: an update of its pharmacology, therapeutic efficacy and tolerability in the treatment of insomnia. Drugs 2000;59:865-889.

2. Rush CR, Baker RW, Wright K. Acute behavioral effects and abuse potential of trazodone, zolpidem and triazolam in humans. Psychopharmacology (Berl) 1999;144:220-233.

3. Merlotti L, Roehrs T, Koshorek G, Zorick F, Lamphere J, Roth T. The dose effects of zolpidem on the sleep of healthy normals. J Clin Psychopharmacol 1989;9:9-14.

4. Langtry HD, Benfield P. Zolpidem. A review of its pharmacodynamic and pharmacokinetic properties and therapeutic potential. Drugs 1990;40:291-313.

5. Salvà P, Costa J. Clinical pharmacokinetics and pharmacodynamics of zolpidem. Therapeutic implications. Clin Pharmacokinet 1995;29:142-153.

6. Korpi ER, Mattila MJ, Wisden W, Lüddens H. GABA(A)-receptor subtypes: clinical efficacy and selectivity of benzodiazepine site ligands. Ann Med 1997;29:275-282.

7. Lancel M. Role of GABAA receptors in the regulation of sleep: initial sleep responses to peripherally administered modulators and agonists. Sleep 1999;22:33-42.

8. Mitler MM. Nonselective and selective benzodiazepine receptor agonists--where are we today? Sleep 2000;23 Suppl 1:S39-S47.

9. Evans SM, Funderburk FR, Griffiths RR. Zolpidem and tri- azolam in humans: behavioral and subjective effects and abuse liability. J Pharmacol Exp Ther 1990;255:1246-1255.

10. Bissell P, Ward PR, Noyce PR. The dependent consumer: reflections on accounts of the risks of non-prescription medicines. Health 2001;5:5-30.

11. Roumie CL, Griffin MR. Over-the-counter analgesics in older adults: a call for improved labelling and consumer education. Drugs Aging 2004;21:485-498.

12. Bush PJ, Rabin DL. Who's using nonprescribed medicines? Med Care 1976;14:1014-1023.

13. Pommer W, Glaeske G, Molzahn M. The analgesic problem in the Federal Republic of Germany: analgesic consumption, frequency of analgesic nephropathy and regional differences. Clin Nephrol 1986;26:273-278.

14. Cooper RJ. 'I can't be an addict. I am. 'Over-the-counter medicine abuse: a qualitative study. BMJ Open 2013;3:e002913.

15. Bryner JK, Wang UK, Hui JW, Bedodo M, MacDougall C, Anderson IB. Dextromethorphan abuse in adolescence: an increasing trend: 1999-2004. Arch Pediatr Adolesc Med 2006; 160:1217-1222.

16. Falck R, Li L, Carlson R, Wang J. The prevalence of dextromethorphan abuse among high school students. Pediatrics 2006; 118:2267-2269.

17. Abbott FV, Fraser MI. Use and abuse of over-the-counter analgesic agents. J Psychiatry Neurosci 1998;23:13-34.

18. Licata SC, Jensen JE, Conn NA, Winer JP, Lukas SE. Zolpidem increases GABA in depressed volunteers maintained on SSRIS. Psychiatry Res 2014;224:28-33.

19. Ghosh S, Bhuyan D. Baclofen abuse due to its hypomanic effect in patients with alcohol dependence and comorbid major depressive disorder. Clin Psychopharmacol Neurosci 2017;15:187-189.

20. Agley J, Gassman R, YoussefAgha A, Jun M, Torabi M, Jayawardene W. Examining sequences of adolescent substance use initiation involving over-the-counter (OTC) drug abuse. J Child Adoles Subst 2015;24:212-219. 